\title{
On Joint Functioning of the Neural Network and Differential Model
}

\author{
Igor F. Yasinskiy* \\ High-performance Computing Systems Department, Ivanovo State Power University, Ivanovo, Russia; \\ igor2266@yandex.ru
}

\begin{abstract}
Objectives: The analytical and numerical solutions of the differential equations systems in practice can be the computationally difficult task. The main objective is to improve the methods of physical processes research, speed up the mathematic model calculations. Methods/Analysis: Neural network training; Software realization of an artificial neural network observes the solution, based on the differential model of the hydrodynamic process and uses the values of channel volume consumption, obtained in the dynamic model, to train the connections weights and carry out its own prediction. Findings: The joining of dynamic model and neural network technology allows synthesizing the adaptive parallel system, which have the significant advantage in computational speed in ccomparisons with the traditional differential conception. Data compression by means of the neural network funnel permits to find the main components in the input data and reduce the time for system training. Application/Improvement: The offered method allows to significantly increasing the speed and quality of the technological object studying. It expands the field of application of such combined systems in automatic control, engineering, in the study of processes described by different types of differential equations.
\end{abstract}

Keywords: Differential Equations, Dynamic Models, Hydrodynamics, Mathematical Modeling, Neural Networks

\section{Introduction}

Research of physical process on the first stage consists in creation of the mathematical model of the process. This model is often represented by the system of the differential equations. The effective analytical and numerical methods are developed for their solution..12 However, in practice these systems can become cumbersome, and solution often demands uunacceptable computing and time expenses. In addition, the application of numerical methods in some cases is being held by the presence of unobserved variables, including their initial values. ${ }^{3}$ One of the perspective methods, which allow solving such a problem, consists in using of combination of the neural network and "classical" differential presentation in the object model.

\section{Hydrodynamic Problem Description}

The proposed ideas were applied in a hydrodynamic problem modeling using a neural network. The unsteady motion of water in open channels is described by the following differential equations (1-6):.,5

$$
\begin{aligned}
& \frac{\partial \omega}{\partial t}+\frac{\partial Q}{\partial x}=q \\
& \frac{\partial Q}{\partial t}+\frac{\partial}{\partial x}\left(P-\frac{Q^{2}}{\omega}\right)=g \omega\left(i-\frac{Q|Q|}{K^{2}}\right)+R_{x}
\end{aligned}
$$

${ }^{*}$ Author for correspondence 
here $Q(x, t)$ - volume consumption, $\omega(x, h)$ - crosssectional area of flow, $h=z(x, t)-z_{0}(x)$ - flow depth, $z(x, t)$ - ordinate of free flow surface, $z_{0}(x)$ - ordinate of channel bottom.

$$
\begin{aligned}
& \omega=\int_{0}^{h} b(x, \xi) d \xi \\
& P=g \int_{0}^{h}(h-\xi) b(x, \xi) d \xi, \\
& R_{x}=g \int_{0}^{h}(h-\xi) \frac{\partial b}{\partial x} d \xi,
\end{aligned}
$$

where $b(x, \xi)$ - width of flow cross section, $i=z_{0}^{\prime}(x)$ - bottom slope, $K(x, h)$ - consumption module, $q(x)$ - ground flow to main stream from shore, $g$ - acceleration of gravity.

$$
K=\omega \cdot C \sqrt{R}
$$

$$
\text { C - coefficient of Chezy, R - hydraulic radius. }
$$

\section{Combination of Neural Network and Dynamic Model}

"Hopes for the construction of high-quality atmospheric models by traditional (dynamic) methods in all likelihood will not come true. Traditional methods lose their effectiveness beyond the "first approximation" and are

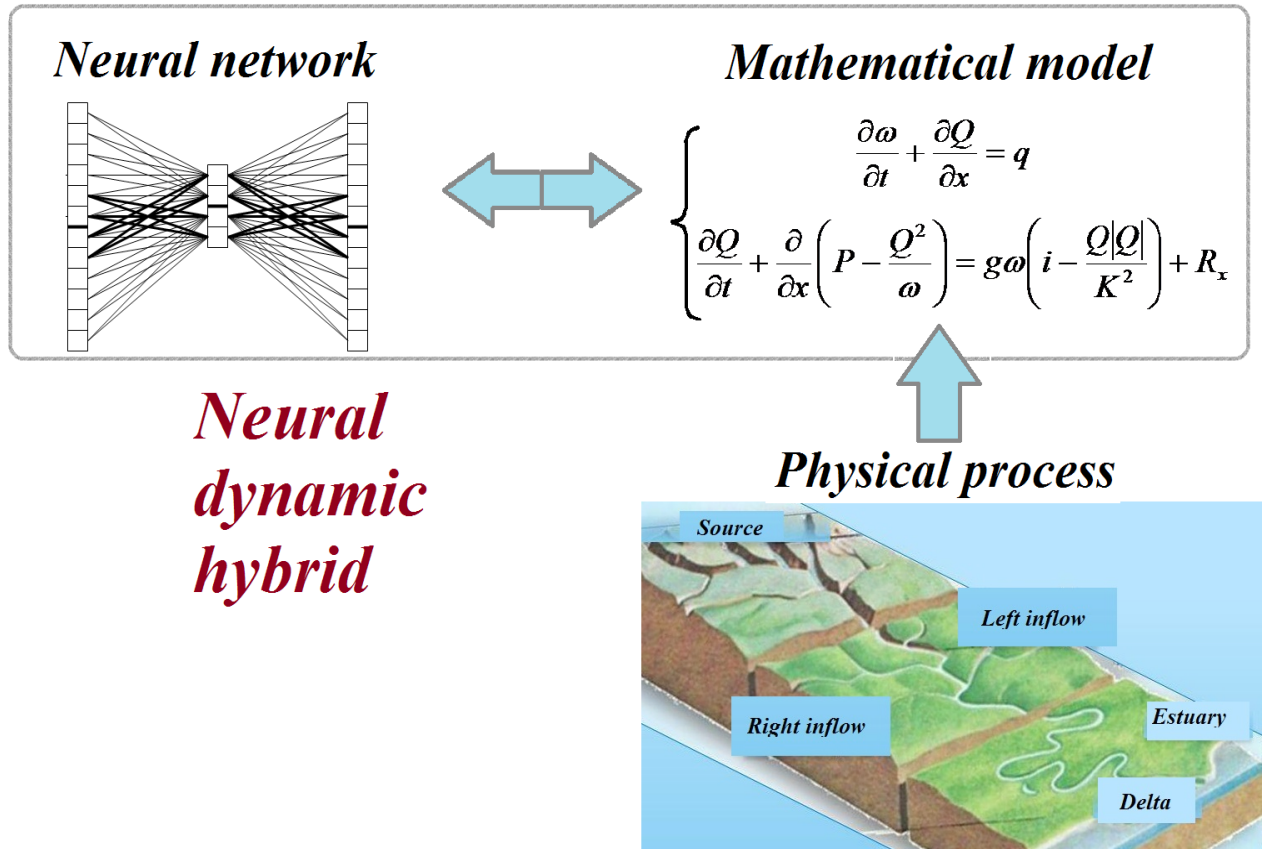

Figure 1. Combined mathematical model, consists of neural network and dynamic model. 
becoming increasingly "cumbersome", “...the only reasonable way out of the impasse created in modern science is the transition to adaptive models, that allows to quickly monitoring the changes in the simulated system. The particular case of the adaptive model is neural network" ${ }^{\prime}$

The offered position differs from the mentioned above. On the basis of computational experience, we believe that the solution should be found on the ways of building of network hybrids, including dynamic and neural network elements shown in Figure 1.

The described method allows combining the neural network prediction with the dynamic one. The process prediction by means of neural network is based on the results of continuous observation of the opened variables behavior. Wherein, the hidden parameters values are not necessary.

First, the neural network observes the dynamic model calculations and learns to predict the process parameters according these calculations. Then, on reaching the desired work quality, the trained neural network may act as a mathematical program model of the object. It can be used for the research of the object properties, definition of the process hidden parameters and its unknown initial conditions.

In the developed computer program the dynamic model, on the basis of the presented differential equations, calculates the volume consumption values $Q(x, t)$

at the grid nodes for a given time interval. At the same time, the software model of an artificial neural network "observes" the process and uses the values $Q(x, t)$

obtained in the dynamic model to adjust the connections weights and carry out its own prediction Figure 1. The neural network input also takes into account the depth of flow parameter $z(x, t)$ and uses the following four-node

schemes shown in Figure 2.

Each input image, thus, consists of $Q$ and $z$ values in a given node, in neighboring nodes by coordinate and in the previous node by time. The network reaction is compared with the $Q[t+t a u, i]$ value. A series of experiments were performed with various learning algorithms. The best results were shown by the variant of back propaga-
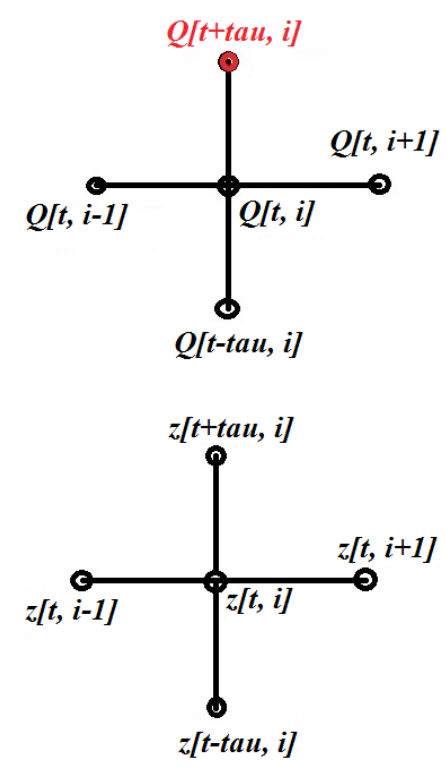

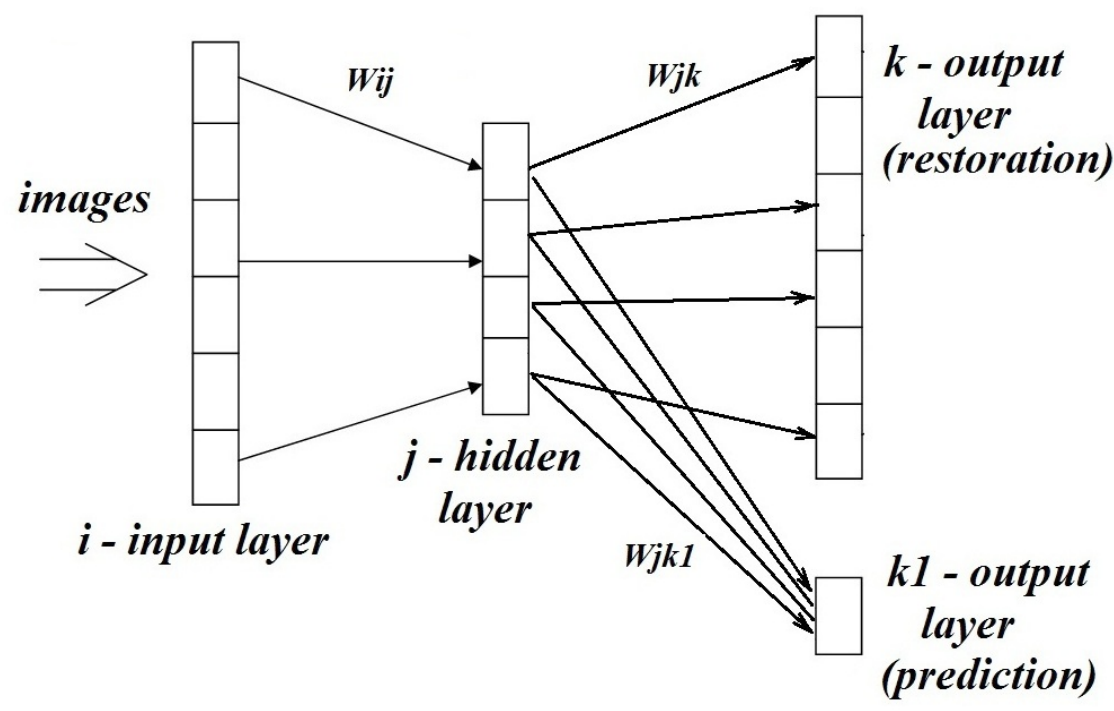

Figure 2. Hydrodynamic model computational schemes, used in neural network teaching and the structure of a neural network with a "funnel" layer. 
tion algorithm, supplemented by procedures of neuron activation according Golovko. ${ }^{7}$ Due to the introduced technique of threshold adjustment, the signal at the neuron input appears on the working site of the activation function, which leads to faster learning. In this case, the forward and reverse trajectories of the back propagation algorithm are written in the following form (7-15).

Direct propagation (7-9):

$$
\begin{aligned}
& O_{j}=F\left(\sum_{i=1}^{N_{i}} W_{j}^{\prime} O_{i}-T_{j}\right), \\
& \mathrm{j}=1,2, \ldots, \mathrm{N}_{\mathrm{j}} \\
& O_{k}=F\left(\sum_{j=1}^{N_{j}} W_{k}^{\prime \prime} O_{j}-T_{k}\right) ; \mathrm{k}=1,2, \ldots \mathrm{N}_{\mathrm{k}} \\
& F(v)=1(1+\exp (-v),
\end{aligned}
$$

where $O_{i}, O_{j} O_{k}$ - neurons output values of the layers $i, j, k$ correspondingly; $F$ - threshold $S$-function of the layers $j$ и $k ; T_{j}, T_{k}$ - parameters, responding for the threshold

adjustment.

The resulting vector on the output $O_{k}, k=1,2, \ldots, N_{k}$ is compared with the required response $Y_{k}$. The error components are calculated (10):

$$
\delta_{k}^{\prime}=Y_{k}-O_{k}
$$

and a back propagation is performed (11-15), during which the weights are adjusted $W_{i j}^{\prime}, W_{j k}^{\prime /}$ :

$$
W_{j k}^{/ / r+1}=W_{j k}^{/ / r}+\eta O_{j} \delta_{k}^{/ /}
$$

$$
\begin{aligned}
& T_{k}^{r+1}=T_{k}^{r}+\eta \delta_{k}^{\prime /}, \\
& \delta_{j}^{\prime}=O_{j}\left(1-O_{j}\right) \sum_{k=1}^{N_{k}} W_{j k}^{\prime /} \delta_{k}^{\prime /}, \\
& W_{i j}^{/ r+1}=W_{i j}^{/ r}+\eta O_{i} \delta_{j}^{\prime}, \\
& T_{j}^{r+1}=T_{j}^{r}+\eta \delta_{j}^{\prime},
\end{aligned}
$$

where the superscripts $r, r+1$ mean the correction number.

During training, the method of information compression by a neural network funnel is used. ${ }^{9}$ The structure of a network with a compressive hidden layer is presented on the Figure 2. It can be seen that the input and output layers of the neural network have the same number of elements. The hidden middle layer - the neck of the neural network funnel - has fewer elements. The funnel performs the function of selecting the most specific important properties inherent in recognized images. Such selection is extremely important in the solution of problems, where the input layer of the neural network receives large amounts of information. The learning process of the described above network consists of two stages. At the first stage, the layer "funnel" is trained. The neural network learns to reproduce with a given accuracy the image of the input layer $i$ on the output layer $k$. In other words, network learns to recover information after compression. For this purpose the weight coefficients $W_{i j}$ (compression) and $W_{j k}$ (restoration) are calibrated. On the second stage the layer $k$, which served for the neural network funnel training, is forgotten and the weights $W_{j k 1}$ are trained for objects recognition. At the same time, the 


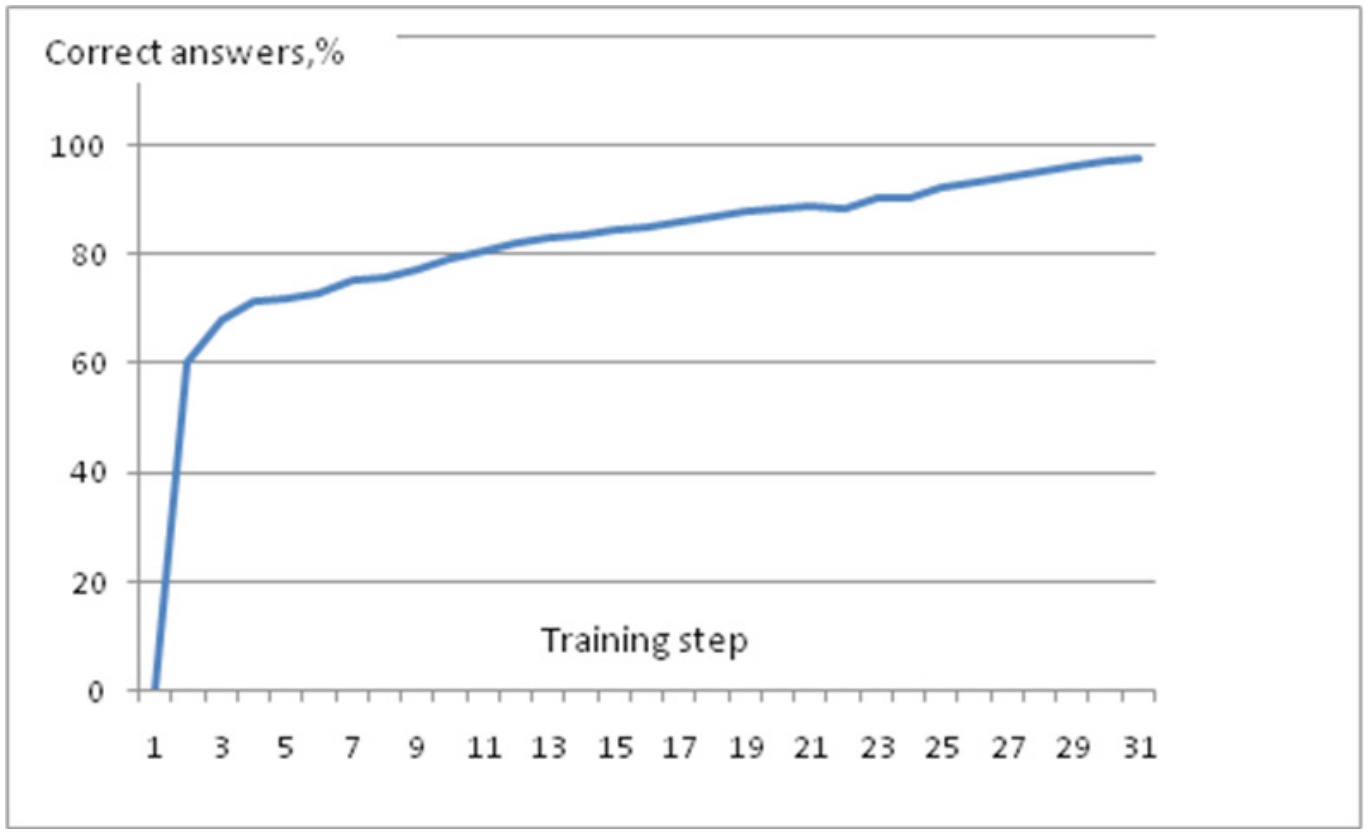

Figure 3. The dependence of the correct answer on the learning step.

weights $W_{i j}$, trained for the information compression, remain unchanged.

\section{Experimental Results}

Numerical experiments showed that such a neural network model of the hydrodynamic process can be quickly trained to high accuracy of prediction. In particular, since the thirtieth step of the training, the difference in the volume consumption $Q(x, t)$ forecast with the help of the

neural network and the dynamic model in all nodes of the spatial grid was less than three percent in Figure 3. Experiments with the computer program also showed a significant increase in the computational speed with the direct progress of the trained neural network in comparison with the dynamic calculations. Under various conditions, the speed advantage was 30 to 90 times.

\section{Conclusion}

A promising direction is the development of combined neural network and dynamic object models. Forecasting processes using neural networks is based on the results of a long-term observation of the behavior of open variables. Addition of a dynamic model by a neural network will allow a more complete and rapid study of the physical processes, determine unknown hidden parameters and initial conditions. Such possibilities will make it possible to use the proposed method in many application areas. 


\section{References}

1. Boas ML. Mathematical methods in the physical sciences. 3rd edition. John Wiley \& Sons Instructors; 2006.

2. Strikwerda JC. Finite difference schemes and partial differential equations. 2nd edition. Madison. University of Wisconsin; 2004. CrossRef. PMid:15461379

3. Tou JT. Modern Control Theory. New York McGraw-Hill; 1964. p. 427.

4. Chow VT. Open-channel hydraulics. New York McGrawHill. 1959; 35(1/28):680. PMCid:PMC2537784

5. Acheson DJ. Elementary fluid dynamics. Oxford University Press; 1990 Aug. p. 408.
6. Sakash IJ, Lankin JP. Neural network forecasting of ozone layer dynamics. Polzunovskiy almanac. 2007; 3(186): 181.

7. Golovko VA. Neural networks training organization and application. Book 10 Moscow IPJR. 2000; 65(4):103247.

8. Callan R. The Essence of Neural Networks. Europe Prentice Hall. 1998. p. 248.

9. Yasinskiy IF. On the information compression by means of the neural network funnel. Technology of the textile industry. 2012; 5(341):154-8. 